\title{
New Proposal for Closed-String Field Theory: The Free-String Case
}

\author{
S.-H. Henry Tye \\ Newman Laboratory of Nuclear Studies, Cornell University, Ithaca, New York 14853
}

(Received 25 May 1989)

\begin{abstract}
A novel form of the free-string field action is proposed for both the closed bosonic string and the heterotic string.

PACS numbers: $11.17 .+y$
\end{abstract}

To study the dynamics and the symmetries of string theory, string field theory seems to be a natural starting point. For this reason, string field theory has attracted a lot of attention since the seventies. More recently, major efforts have gone into the covariant formulation of string field theory. Following Siegel's suggestion, ${ }^{1}$ the covariant action for the open bosonic string field theory was written down by Witten ${ }^{2}$ and investigated by many groups. ${ }^{3-6}$ However, the covariant formulation of closed string field theory turns out to be much more difficult than expected. In terms of the operator formalism of string theory, open strings and closed strings are very closely related. Hence many attempts of closed string field theory formulations are various extensions of the open-string formulation. So far, none of the covariant formulations proposed can reproduce both the four-point scattering amplitude and the one-loop diagrams, which are necessary requirements for a consistent formulation. Since the heterotic string offers the only hope to describe nature and it is a closed-string theory, it is important to continue the search for the covariant formulation of closed-string field action.

The difficulty of the covariant formulation of closedstring field theory can be seen in the following way. Given any formulation, one may use it to calculate both the four-point scattering amplitude and the one-loop diagrams. All formulations proposed so far invariably failed to reproduce the known results, in particular, the covering of the moduli, as discussed clearly by Zweibach. ${ }^{7}$ It is well known that the moduli are associated with the propagators; i.e., the presence of each propagator in a diagram introduces a complex modulus and there are no moduli associated with the vertices. This suggests that the propagator is incorrect, and hence the kinetic term in every formulation of closed-string field theory proposed so far needs modification. In fact, after gauge fixing, all proposals yield essentially the same propagator; hence, it is not surprising that all of them fail.

Let us examine the reason for the failure in more detail. To be specific, let us concentrate on the one-loop diagram, say, the partition function or the one-loop dilaton tadpole diagram. It is well known that the integration region is the fundamental domain in the complex $\tau$ plane. In the various formulations examined in Ref. 7, the propagator essentially involves a projection operator $P$ :

$\Delta=\frac{b_{0} \bar{b}_{0} P}{L_{0}+\bar{L}_{0}}=b_{0} \bar{b}_{0} \int_{0}^{1} d \rho \rho^{L_{0}+\bar{L}_{0}-1} \int_{-1 / 2}^{1 / 2} d \theta e^{2 \pi i \theta\left(L_{0}-\bar{L}_{0}\right)}$,

where $b_{0}\left(\bar{b}_{0}\right)$ are the antighost zero mode for the left (right) mover. In terms of the complex modulus $\tau=\left(\tau_{1}, \tau_{2}\right), \rho=\exp \left(-\tau_{2}\right)$. For example, a typical kinetic energy term in the free-string field theory ${ }^{8}$ which gives the above propagator is given by

$$
\begin{aligned}
S & =\int d X d b d c \Phi K \Phi \\
& =\int d X d b d c \Phi\left(c_{0} \bar{Q}+\bar{c}_{0} Q\right) P \Phi,
\end{aligned}
$$

where $P$ is the same projection operator in Eq. (1), $Q(\bar{Q})$ is the Becchi-Rouet-Stora-Tyutin (BRST) operator for the left (right) mover, and $c_{0}\left(\bar{c}_{0}\right)$ is the zero ghost mode for the left (right) mover. $\Phi$ is the closedstring field. Note that $Q^{2}=\bar{Q}^{2}=0$. After gauge fixing to the Siegel gauge, one obtains the propagator in Eq. (1). There are clear problems with this propagator. It vanishes whenever the left-right levels do not match (i.e., when the $L_{0}$ value differs from the $\bar{L}_{0}$ value), independent of the value of $\tau_{2}$. In particular, the integrations of $\tau_{1}$ and $\tau_{2}$ factorize; i.e., the domain of integration of $\tau_{1}$ is independent of $\tau_{2}$. This means the boundaries of the integration region are straight lines parallel to the $\tau_{1}$ or $\tau_{2}$ axis in the $\tau$ complex plane. However, the fundamental domain in the complex $\tau$ plane has a boundary which correlates $\tau_{1}$ and $\tau_{2}$, namely, $|\tau|=1$. Hence, the partition function following from Eq. (1) cannot have the correct covering of moduli. A priori, it is still possible that, in closed-string field theory, the one-loop tadpole is the physically relevant quantity, not the partition function. However, a careful analysis in Ref. 7 shows that the one-loop tadpole diagram using the above propagator cannot give the correct covering of the moduli, independent of the details of the form of the interaction vertex.

On shell, the projection $P$ provides the necessary level matching for physical states. However, off shell, the projection operator $\boldsymbol{P}$ seems to prevent the correct covering of the moduli. It is natural to ask if there exists a propa- 
gator which has different off-shell properties from that given in Eq. (1), and what is the corresponding kinetic term in string field theory. Of course, this new kinetic term must reproduce the correct physical spectrum. In this paper, such an alternative form is proposed. Although the interaction vertex remains to be constructed and the covering of the moduli remains to be checked, it is already interesting that a totally different kinetic term can be written down. As we shall explain, the motivation for this new form actually comes from an examination of the properties of tree $N$-point scattering amplitudes. In fact, the properties of the scattering amplitudes provide some guidance on the form of the interaction vertex.

The free closed-bosonic-string field action is given by

$$
S=\int \frac{1}{2} \Phi K \Phi,
$$

where

$$
K=\frac{2 \pi Q \bar{Q}}{\sin \left[\pi\left(L_{0}+\bar{L}_{0}\right)\right]} .
$$

Since this action is quite novel, let us explain some of its properties. The action is obviously invariant under the gauge transformation $\delta \Phi=Q \Lambda+\overline{Q \Lambda}$ since $Q, \bar{Q}, L_{0}$, and $\bar{L}_{0}$ all (anti)commute, and $Q^{2}=0, \bar{Q}^{2}=0$. Hence, we can go to the Seigel-type gauge where $b_{0} \Phi=\bar{b}_{0} \Phi=0$. The gauge-fixed equation of motion is

$$
\hat{K} \Phi=\frac{2 \pi c_{0} \bar{c}_{0} L_{0} \bar{L}_{0}}{\sin \left[\pi\left(L_{0}+\bar{L}_{0}\right)\right]} \Phi=0 .
$$

Recall that $L_{0}=p^{2} / 2-N$ and $\bar{L}_{0}=p^{2} / 2-\bar{N}$, where $N$ $(\bar{N})$ is the left (right) mover excitation number and $\mathbf{p}$ is the momentum. Consider the case when $L_{0}$ is approaching zero while $\bar{L}_{0}=p^{2} / 2-\bar{N}$; then $p^{2} / 2 \rightarrow N$ while $\bar{L}_{0} \rightarrow N-\bar{N}$, so

$$
\sin \left[\pi\left(L_{0}+\bar{L}_{0}\right)\right] \rightarrow(-1)^{N-\bar{N}} 2 \pi\left(\frac{p^{2}}{2}-N\right)
$$

so that the zero from $L_{0}$ is canceled by the zero from the denominator. Therefore $\hat{K} \Phi=0$ if and only if $L_{0} \Phi$ $=\bar{L}_{0} \Phi=0$. Hence, on shell, Eq. (5) will reproduce the correct spectrum. It is possible that $K$ has an extra phase factor $\exp \left[i \pi\left(L_{0}+\bar{L}_{0}\right)\right]$.

The corresponding gauge-fixed propagator is given by

$$
\Delta^{\prime}=\frac{b_{0} \bar{b}_{0} \sin \left[\pi\left(L_{0}+\bar{L}_{0}\right)\right]}{2 \pi L_{0} \bar{L}_{0}} .
$$

The pole structures of this propagator are identical to that of the standard propagator in Eq. (1). However, their off-shell properties are very different; in particular, the propagator in Eq. (1) vanishes for off-shell states $N \neq \bar{N}$ while the new propagator in Eq. (7) does not vanish. On the other hand, the new propagator has zeros whenever $p^{2}$ hits integer values, due to the sine factor. Note that, in the $s$ channel, $\sin (\pi s)=\sin \left[\pi\left(L_{0}\right.\right.$ $\left.\left.+\bar{L}_{0}\right)\right](-1)^{N+\bar{N}}$ for any state in the system.
It is important to point out that the new propagator given in Eq. (7) is motivated by an examination of closed-string tree amplitudes. Recall that a general $\mathrm{N}$ point closed-string tree amplitude can be written as a finite sum of a product of two open-string tree amplitudes. ${ }^{9}$ For example, let us consider the four-point amplitude, which clearly illustrates our point,

$$
A^{\text {closed }}(s, t, u)=\sin (\pi s) A_{\text {left }}^{\text {open }}(s, t) A_{\text {right }}^{\text {open }}(s, u) \text {, }
$$

where $A_{\text {left }}^{\text {open }}(s, t)$ has poles in $(s, t)$ channels while $A_{\text {right }}^{\text {open }}(s, u)$ has poles in $(s, u)$ channels; the double poles in the $s$ channel are precisely canceled by the $\sin (\pi s)$ factor. For $N$-point amplitude with $N-3$ propagators, there are naturally $N-3$ sine factors to remove possible double poles in the $1 / L_{0} \bar{L}_{0}$ factors coming from the operator formalism of the corresponding open-string amplitudes for left and right movers. For a heterotic string, $A_{\text {left }}^{\text {open }}(s, t)$ is bosonic and $A_{\text {right }}^{\text {open }}(s, u)$ is supersymmetric in Eq. (8).

The new kinetic term is nonlocal. It is precisely this nonlocal property that results in the zeros in the amplitudes whenever $\sin (\pi s)$ vanishes. Note that the vertex for left movers $V_{L}$ and the vertex for right movers $V_{R}$ must have a relative $\operatorname{SL}(2, C)$ transformation so that the cross channels have only single poles. This $\operatorname{SL}(2, C)$ transformation is closely related to the twist operator introduced long ago. ${ }^{10}$ This imposes a stringent condition on the construction of the interaction term.

The closed-string field $\Phi$ can be defined in terms of a direct product of two open-string fields:

$$
\Phi=\psi_{\text {left }}\left(x_{\mu}, \partial X_{\mu}, b, c\right) \psi_{\text {right }}\left(x_{\mu}, \bar{\partial} X_{\mu}, \bar{b}, \bar{c}\right),
$$

where $\psi_{\text {left }}$ is defined in Refs. 4 and 6 in the operator formulation for the open-bosonic-string field theory, where $\partial X_{\mu}, b$, and $c$ are functions of the complex variable $z$. Similarly $\psi_{\text {right }}$ can be defined, except the variables are now functions of $\bar{z}$. For any given tensor field $f(z)$ of dimension $d$, let us define

$$
f_{n}=\oint \frac{d z}{2 \pi i} z^{n+d-1} f(z)
$$

and similar definitions for the antiholomorphic fields. Now we can introduce the Fourier modes $\alpha_{n}, b_{n}, c_{n}, \bar{\alpha}_{n}$, $\bar{b}_{n}$, and $\bar{c}_{n}$, where $\partial X, b$, and $c$ have dimensions 1,2 , and -1 , respectively. The only common coordinates in $\psi_{\text {left }}$ and $\psi_{\text {right }}$ are the position $x_{\mu}$ and the momentum operator $\alpha_{0}=\bar{\alpha}_{0}$.

Let us define the $\operatorname{SL}(2, C)$ vacuum $|0\rangle$ to have zero ghost number. ${ }^{6}$ Then the ground state $|\Omega\rangle=\bar{c}_{1} c_{1}|0\rangle$ and the momentum eigenvector is given by $|p\rangle$ $=\exp [i p X(0)]|\Omega\rangle$, where $\alpha_{0}|p\rangle=p|p\rangle$. Now, we can write out explicitly the string field $\Phi$, whose total ghost number is 2 units. In momentum space it is

$$
\begin{aligned}
\Phi= & \Phi(1,1)+\Phi(2,0)+\Phi(0,2) \\
& +\Phi(3,-1)+\Phi(-1,3)+\cdots,
\end{aligned}
$$


where $\Phi(j, 2-j)$ has a ghost number $j$ for the left mover and $2-j$ for the right mover;

$$
\begin{aligned}
& \Phi(1,1)=\left\{s(p)+G_{\mu v}(p) \alpha_{-1}^{\mu} \bar{\alpha}_{-1}^{v}+U_{\mu}(p) \bar{\alpha}_{-1}^{\mu} c_{0} b_{-1}+V_{\mu}(p) \alpha_{-1}{ }_{1} \bar{c}_{0} \bar{b}_{-1}+w(p) c_{0} b_{-1} \bar{c}_{0} \bar{b}_{-1}+\cdots\right\}|p\rangle, \\
& \Phi(2,0)=\left\{\eta(p) c_{-1} \bar{b}_{-1}+A_{\mu}(p) c_{0} \alpha_{-1}^{\mu} \bar{b}_{-1}+\cdots\right\}|p\rangle, \\
& \Phi(0,2)=\left\{\sigma(p) b_{-1} \bar{c}_{-1}+B_{\mu}(p) b_{-1} \bar{\alpha}_{-1}^{\mu} \bar{c}_{0}+\cdots\right\}|p\rangle,
\end{aligned}
$$

and so on. Here $s(p)$ is the tachyon; $G_{\mu v}(p)$ contains the graviton and the antisymmetric tensor field. Fields whose left and right levels do not match may also be included in the above formulas. Since the BRST operator has unit ghost number, and the conformal matrix elements vanish unless they have 3 units of ghost number for both the left and the right movers, the action becomes

$$
S=\pi \int \Phi(1,1) \frac{Q \bar{Q}}{\sin \left[\pi\left(L_{0}+\bar{L}_{0}\right)\right]} \Phi(1,1)+\Phi(2,0) \frac{Q \bar{Q}}{\sin \left[\pi\left(L_{0}+\bar{L}_{0}\right)\right]} \Phi(0,2)+\cdots
$$

It is interesting to work out explicitly the kinetic term for the massless sector. Let us consider for the moment the first term. Keeping only up to quadratic power in momentum $p$, it is straightforward to show that all fields in Eq. (11) except $G_{\mu v}(p)$ are auxilliary fields and can be integrated out. Besides the usual kinetic energy terms for the antisymmetric tensor field $B_{\mu v}(p)$ and the graviton $\tilde{h}_{\mu v}(p)$, we also have a nonlocal term corresponding to the linearization of $\int R\left(\partial^{2}\right)^{-1} R$, where $R$ is the Riemann scalar in Einstein theory. It is known that in string theory, the dilaton field $D(p)$ couples to $R(p)$. Integrating out the dilaton field introduces such a nonlocal term. ${ }^{11}$ Where is the dilaton field $D(p)$ ? The only other term in the action $S$ that contributes to the massless sector is the second term in Eq. (13) where the string fields $\Phi(2,0)$ and $\Phi(0,2)$ are given in Eq. (12). Here $A_{\mu}$ and $B_{\mu}$ are auxilliary fields while the fields with a kinetic term take the form $\sigma p^{2} \eta$. Integrating out the auxilliary fields precisely cancels out the above term and no dynamical field is left. However, if we now demand that $\tilde{h}_{\mu \mu}$ and the fields in Eq. (12) mix in a way such that no nonlocal term is left behind after all auxilliary fields have been integrated out, we obtain the standard action for $B_{\mu v}$, the graviton $h_{\mu v}$, and the dilaton $D(p)$, where $[f=(\sigma+\eta) / \sqrt{2}]$

$$
\begin{aligned}
& h_{\mu v}=\tilde{h}_{\mu v}+\left(\delta_{\mu v}-\frac{p_{\mu} p_{v}}{p^{2}}\right) f, \\
& D=f+\left(\delta_{\mu v}-\frac{p_{\mu} p_{v}}{p^{2}}\right) \tilde{h}_{\mu v} .
\end{aligned}
$$

The kinetic energy term for heterotic string field theory is also easy to write down. Borrowing from Witten's formulation for open superstrings, ${ }^{12}$

$$
K=\frac{2 \pi Q_{\text {bosonic }} \bar{Q}_{\text {superstring }}}{\sin \left[\pi\left(L_{0}+\bar{L}_{0}\right)\right]}
$$

and $\Phi=\phi_{\text {left }} \phi_{\text {right }}$, where $\phi_{\text {right }}=(a, \Psi)$ as given in open superstring. Here $a$ is the Neveu-Schwarz field and $\Psi$ is the Ramond field. For the right mover, an inverse picture-changing operator $Y$ must be inserted for the Ra- mond sector $\Psi .{ }^{12}$

The construction of the interaction term is technically more difficult, and is under investigation. The first step is to generalize the linear gauge transformation to nonlinear gauge transformation and then demand closure of the gauge transformations. This step can be achieved; however, to obtain the correct interaction terms remains to be seen. In summary, we find it intriguing that the kinetic term of closed-string fields can be written in a nonlocal form. In fact, it was argued by Woodard ${ }^{13}$ that a nonlocal kinetic operator is necessary for the correct loop diagrams. Of course, as discussed in Ref. 14, a proper formulation of string field theory probably requires an enlarged configuration space for string fields. However, the construction described here can be generalized easily for the new configuration space.

I thank Jacques Distler, Zvonimir Hlousek, Hikaru Kawai, David Lewellen, Edwin Lyman, and Zongan Qiu for discussions. This research was supported in part by the National Science Foundation under Contract No. PHY-82-09011.

\footnotetext{
${ }^{1}$ W. Siegel, Phys. Lett. 151B, 391 (1985); 151B, 396 (1985); M. Kato and K. Ogawa, Nucl. Phys. B212, 443 (1983).

${ }^{2}$ E. Witten, Nucl. Phys. B268, 253 (1986).

${ }^{3}$ S. Giddings, Nucl. Phys. B278, 242 (1986); S. Giddings and E. Martinec, Nucl. Phys. B278, 91 (1986); S. Giddings, E. Martinec, and E. Witten, Phys. Lett. B 176, 362 (1986).

${ }^{4}$ D. Gross and A. Jevicki, Nucl. Phys. B B282, 1 (1987); B287, 362 (1987); E. Cremmer, C. B. Thorn, and A. Schwimmer, Phys. Lett. B 179, 57 (1986).

${ }^{5}$ K. Itoh, T. Kugo, H. Kunitomo, and H. Ooguri, Prog. Theor. Phys. 75, 162 (1986); H. Hata, K. Itoh, T. Kugo, H. Kunitomo, and K. Ogawa, Phys. Rev. D 34, 2360 (1986); 35, 1318 (1987); W. Siegel and B. Zweibach, Nucl. Phys. B263, 105 (1985); T. Banks and M. E. Peskin, Nucl. Phys. B264, 513 (1986).

${ }^{6}$ A. LeClair, M. E. Peskin, and C. R. Prietschopf, Nucl. Phys. B317, 411 (1989); B317, 464 (1989).

${ }^{7}$ B. Zweibach, Ann. Phys. (N.Y.) 186, 111 (1988).

${ }^{8}$ A. Neveu, H. Nicolai, and P. C. West, Nucl. Phys. B264,
} 
173 (1986); J. Lykken and S. Raby, Nucl. Phys. B278, 256 (1986).

${ }^{9}$ H. Kawai, D. C. Lewellen, and S.-H. H. Tye, Nucl. Phys. B269, 1 (1986).

${ }^{10}$ L Caneschi, A. Schwimmer, and G. Veneziano, Phys. Lett. 30B, 351 (1969); D. Amati, M. LeBellac, and D. Olive, Nuovo Cimento 66A, 831 (1970).

${ }^{11}$ See, e.g., M. E. Peskin, in Proceedings of the Theoretical Advanced Study Institute in Elementary Particle Physics,
1986, edited by H. E. Haber (World Scientific, Singapore, 1987).

${ }^{12}$ E. Witten, Nucl. Phys. B276, 291 (1986); D. Gross and A. Jevicki, Nucl. Phys. B243, 29 (1987); A. LeClair and J. Distler, Nucl. Phys. B273, 552 (1986); J. P. Yamron, Phys. Lett. B 187, 67 (1987).

${ }^{13}$ R. P. Woodard, Phys. Lett. B 213, 144 (1988).

${ }^{14} \mathrm{Z}$. Qiu and S.-H. H. Tye, Cornell University Report No. CLNS 89/919, 1989 (unpublished). 